\title{
State-of-the-Art Flash Chips for Dosimetry Applications
}

\author{
Preeti Kumari, Levi Davies, Narayana P. Bhat, En Xia Zhang, Senior Member, IEEE, Michael W. \\ McCurdy, Senior Member, IEEE, Daniel M. Fleetwood, Fellow, IEEE, and Biswajit Ray, Member,
} IEEE

\begin{abstract}
In this paper we show that state-of-the-art commercial off-the-shelf Flash memory chip technology $(20 \mathrm{~nm}$ technology node with multi-level cells) is quite sensitive to ionizing radiation. We find that the fail-bit count in these Flash chips starts to increase monotonically with gamma or $\mathrm{X}$-ray dose at $100 \operatorname{rad}\left(\mathrm{SiO}_{2}\right)$. Significantly more fail bits are observed in X-ray irradiated devices, most likely due to dose enhancement effects due to high- $Z$ back-end-of-line materials. These results show promise for dosimetry application.
\end{abstract}

\section{INTRODUCTION}

UTONOMOUS, real-time radiation sensing with high $\mathbf{A}_{\text {precision is an important topic for several applications }}$ including military, civilian health and safety, spaceexploration, etc. There exist different types of radiation dosimeters, such as small finger/body film badges, thermoluminescent dosimeters (TLDs) [1], optically stimulated luminescence dosimeters (OSLs) and electronic field effect transistor based dosimeters (RADFETs) [2]-[5], each with its own strengths and weaknesses [6]. Many types of dosimeters need to be sent to a laboratory to be evaluated for exposure level and hence cannot perform real-time in situ measurement of the radiation environment.

Flash memory chips are low cost, high density with smallfootprint and widely used in many embedded systems such as smartphones, etc. Flash chips store information in the form of charge on its floating gate (see Fig. 1(a)). A floating gate metal-oxide-semiconductor field effect transistor (FGMOSFET), allows electrons from the silicon substrate to tunnel into the floating gate during the program operation. Under ionizing radiation exposure, charge loss takes place from the floating gate of the Flash cell through the mechanisms [7]-[10] shown in Fig 1(b). Charge loss decreases the programmed cell threshold voltage $\left(\mathrm{V}_{\mathrm{t}}\right)$ distribution (see Fig. 1(c)), resulting in " 0 " $\rightarrow$ " 1 " fails [11][13].

Manuscript received June 28, 2018.

Preeti Kumari, Levi Davies, and Biswajit Ray are with the Electrical and Computer Engineering Department, University of Alabama in Huntsville, Huntsville, AL 35899 USA (e-mail: biswajit.ray@uah.edu).

Narayana P. Bhat is with The Center for Space Plasma and Aeronomic Research, University of Alabama in Huntsville, Huntsville, AL 35899 USA (e-mail: bhatn@uah.edu).

En Xia Zhang, Mike McCurdy, and Daniel M. Fleetwood are with the Electrical Engineering and Computer Science Department, Vanderbilt University, Nashville, TN 37235 USA (e-mail: enxia.zhang@ vanderbilt.edu, mike.mccurdy@vanderbilt.edu and dan.fleetwood@vanderbilt.edu).
The key idea behind Flash-based dosimetry is to correlate the radiation induced fail bit count $(\mathrm{FBC})$ with the radiation dose [6]-[9]. In 1998 Scheick et al. [14] demonstrated the utility of Electrically Erasable Programmable Read-Only Memory (EEPROM) for measuring ionizing radiation in space through the Microelectronics and Photonics Test Bed (MPTB) satellite. More recently, Savage et al. [15] proposed extreme value analysis in order to use floating gate memory as a dosimeter. In this paper we use multiple Flash chips of $20 \mathrm{~nm}$ technology node from Micron Technology in order to demonstrate its usability as a dosimeter. Our key contributions in this paper are as follows:

1) We find that commercial un-modified MLC (multi-level cell) Flash chips can detect radiation doses as low as 100 $\operatorname{rad}\left(\mathrm{SiO}_{2}\right)$ during $\mathrm{X}$-ray and gamma-ray exposure.

2) We propose and demonstrate different techniques in order to improve radiation sensitivity and minimize measurement errors.

3) We propose an algorithm for the development of a smart phone application on the Android platform based on the radiation response of Flash characteristics in order to demonstrate the possibility of continuous real-time monitoring of the radiation dose.

\section{EXPERIMENTAL DETAILS}

We have utilized commercial off the shelf NAND Flash memory chips of $20 \mathrm{~nm}$ technology node from Micron Technology (part \# MT29F64G08CBABAWP: B TR) in TSOP (Tape and Reel) packages. The chips are of size $64 \mathrm{~Gb}$ with MLC storage. In the experiments we have used 100 different blocks from different physical locations of the chip. Each block consists of 256 pages of size $8 \mathrm{k}$ bytes each. A custom-designed board is used in order to program and read the Flash chips. The board contains a socket to hold a Flash chip under test, an ARM microprocessor to issue commands and receive data from the Flash chip, and a serial interface.

X-ray irradiation was performed using an ARACOR Model $410010-\mathrm{keV} \mathrm{X}$-ray irradiator at a dose rate of $5 \mathrm{krad}\left(\mathrm{SiO}_{2}\right)$ / min. All the terminals of the device under test (DUT) were grounded during exposure. Gamma-ray experiments were performed using a Cs-137 isotopic irradiator at a dose rate of $20 \mathrm{rad}\left(\mathrm{SiO}_{2}\right) / \mathrm{min}$. Both X-ray and Gamma-ray exposures [16] were performed in the radiation test facilities of Vanderbilt University. 


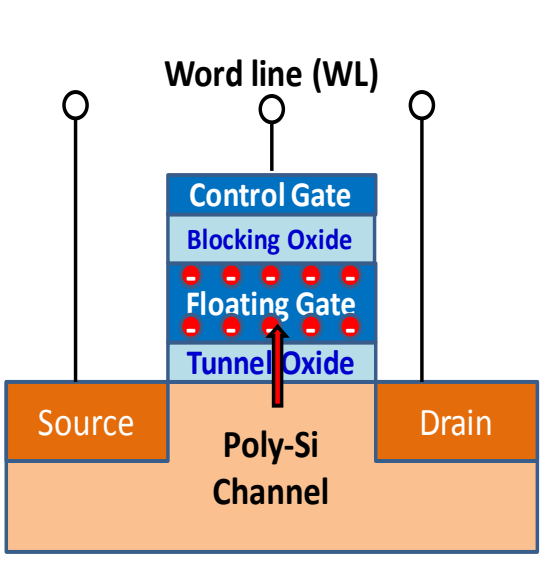

(a)



(b)

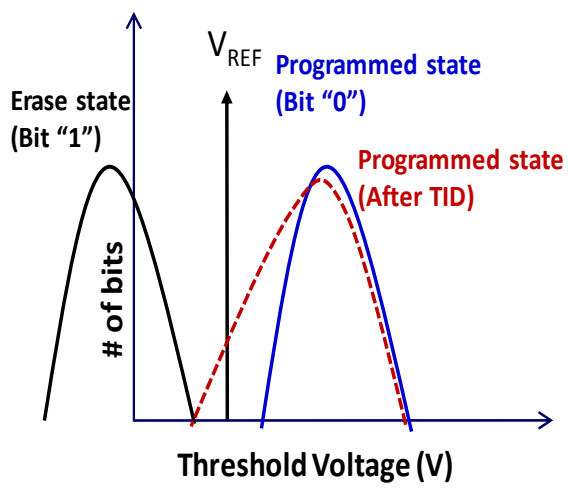

(c)

Fig. 1. (a) Schematic diagram of a floating gate Flash memory cell. (b) Energy band diagram with different possible pathways for charge loss after irradiation: (1) electron emission through the tunnel oxide and blocking oxide, (2) trap assisted tunneling, (3) generation of electron-hole pairs in blocking/tunnel oxide and subsequent recombination or hole trapping, and (4) conductive pipe model for charge loss. (c) Threshold voltage $\left(V_{t}\right)$ distribution of the memory cells in a page/block. After ionizing radiation exposure, the program state $\mathrm{V}_{\mathrm{t}}$ shifts, causing fail bits. (After [7]-[13].)

\section{RESUlTS AND ANALYSIS}

In order to evaluate the effects of X-ray exposure on the Flash chips, we first remove the packaging material from the TSOP chip, as shown in Figs. 2(a) and (b). We then program the entire chip with an "ALL-0" data pattern. Just after the program, if we read the chip back, we find that there are a few bits already in the failed state ("0" $\rightarrow$ " 1 " bit flip shown as blue dots in Fig. 2(c)). These fail bits are inherent in the MLC (multi-level-cell) chips of advanced technology nodes due to very minimal voltage margin between the programmed states [17]. This inherent fail bit count (FBC) in a page of $8 \mathrm{k}$ bytes size is very small ( $<10$ bits per page), and can be easily corrected by the standard error correction (ECC) engine. The inherent FBC remains almost the same or slightly increases (over a few months of time) at room temperature. Once the chip is exposed to ionizing radiation (X-ray in the case of Fig. 2 ), the FBC increases significantly as a function of radiation dose, as shown in Fig. 2(c). Each data point in Fig. 2(c) is the fail bit count per page (page number shown on $\mathrm{X}$-axis) after radiation exposure. We find that the radiation induced $\mathrm{FBC}$ increase varies from page to page within a block, as shown in the scatter plot in Fig. 2(c). This intra-block variation is due to the inherent differences between the pages within a block. However, the block average FBC remains approximately the same for all the blocks of the same chip (see Fig. 3(a)), which is irradiated uniformly. This implies that, for the purpose of radiation dosimetry, reading of a few blocks in a chip may provide sufficient accuracy.

In Fig. 3(b) we show the cumulative probability distribution function (CDF) for $\mathrm{FBC} /$ page in the chip as a function of X-ray dose. The steepness of the CDF curves indicates minimal variation in the measured block-averaged FBC. In Fig. 3(c) we plot the functional relationship between average FBC and the X-ray radiation dose. These results can be used for calibration purposes for the Flash based dosimetry for the X-rays. In the same plot we also compare the radiation response of the Flash chip with and without its package (or capping). The chip is more sensitive to X-ray irradiation without any capping on it. This is because the packaging material blocks most of the $\sim 10-\mathrm{keV}$ X-rays, allowing only the high energy X-rays in the tail of the distribution to penetrate inside the package [18].

We have also performed experiments in order to quantify the total ionization response using gamma rays (Cs-137 isotopic irradiator). With gamma rays we were able to control the expose the device in dose steps of $100 \mathrm{rad}\left(\mathrm{SiO}_{2}\right)$. In Fig. 4 we plot the evaluation results for the effects of gamma-rays on the Flash chip of the same specification. Similar to the X-

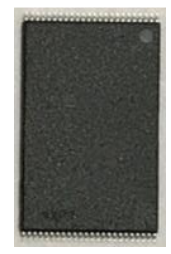

(a)

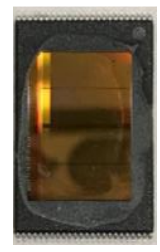

(b)

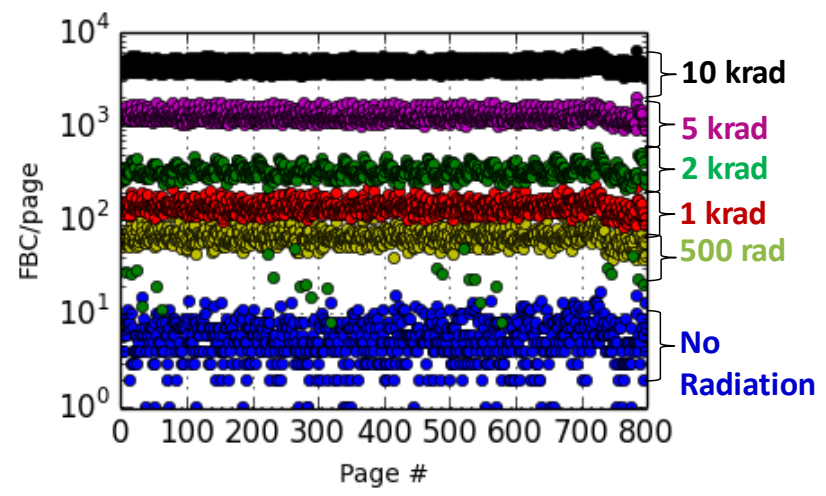

(c)

Fig. 2. (a) Flash memory chip with packaging on. (b) De-capped Flash chip. (c) Scatter plot of fail bits on different pages of the same chip as a function of radiation (X-ray) dose. 


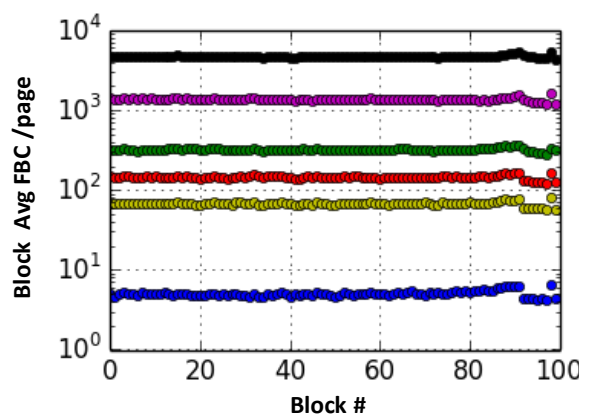

(a)

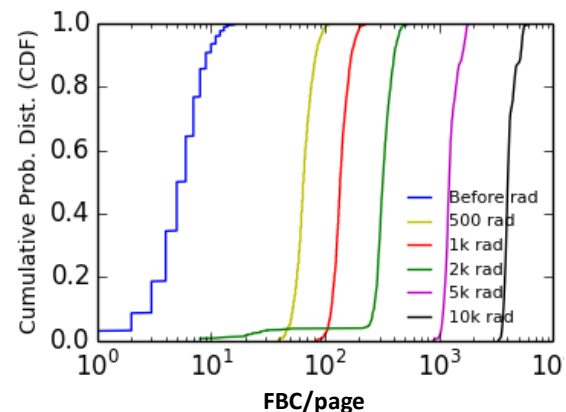

(b)

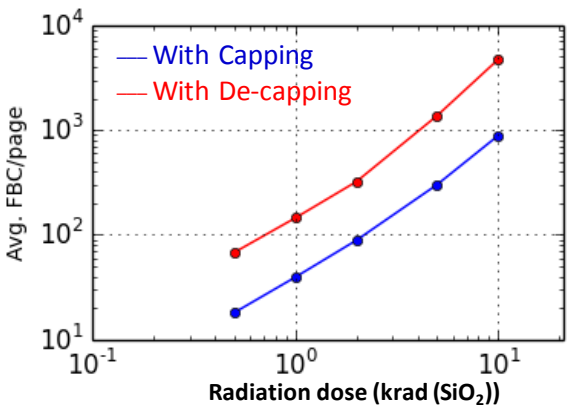

(c)

Fig. 3. Fail bit count for X-ray exposure of a Flash memory chip. (b) Block average FBC/page for each block is plotted as a function of radiation dose. The color coding for the dose levels is the same as in (a). (c) The cumulative probability distribution of the FBC for each radiation dose level is plotted. The legend in the plot shows the dose level. The FBC value just after programing is very similar in both chips.



(a)

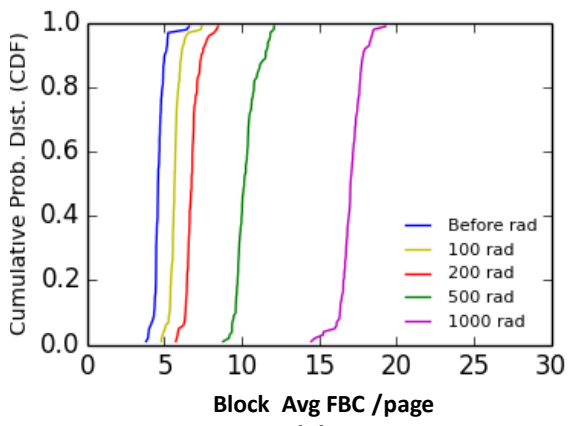

(b)



(c)

Fig. 4. Fail bit count for gamma-ray exposure of a Flash memory chip. (a) Increase in FBC with radiation dose. (b) The cumulative probability distribution of the FBC for each radiation dose level. (c) Average FBC in a chip as a function of radiation dose starting with $100 \operatorname{rad}\left(\mathrm{SiO}_{2}\right)$.

ray exposure, the FBC on the freshly written chip increases with the increase of gamma-ray dose.

Note in Fig. 4 that the MLC chip is sensitive enough to show radiation damage even with $100 \operatorname{rad}\left(\mathrm{SiO}_{2}\right)$ of exposure, which can be measured with standard digital interfaces. These results imply that the state of the art Flash chip with lower technology nodes are quite sensitive to total-ionizingdose effects. This limits their potential use in space environments, but is promising for low intensity radiation dosimetry.

The fail bit count in the $\mathrm{X}$-ray exposure is significantly higher than in the gamma ray exposure for the same dose. For example, $\sim 120 \pm 10 \mathrm{FBCs}$ are observed in Fig. 3 for X-ray irradiation to $1 \mathrm{krad}\left(\mathrm{SiO}_{2}\right)$, while only $17 \pm 1 \mathrm{FBCs}$ are observed for gamma-ray irradiation to $1 \mathrm{krad}\left(\mathrm{SiO}_{2}\right)$ in Fig. 4. The higher FBC during X-ray irradiation is due most likely to dose enhancement effects [16], [19]-[21] associated with the presence of high-Z materials (e.g., W) that are typically present in the back end of the line (BEOL) layers of state-ofthe art memory chips [22]-[24]. Dose enhancement effects of up to 5-10 times can be observed for devices with similar high-Z BEOL materials irradiated with $10-\mathrm{keV}$ X-rays [18],[25], consistent with these results. More work is required to understand and quantify these effects for different types of radiation sources.

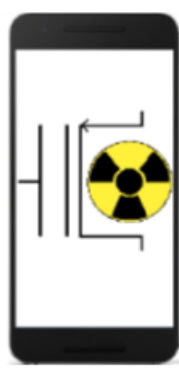

(a)



Fig 5. (a) A prototype for radiation dosimetry application. (b) Algorithm for reporting radiation alert by the application.

Since Flash memory is frequently used in modern smartphone devices, the results in this paper encourage us to develop an application program for smart-phones that can autonomously record the radiation exposure. In Fig. 5 we propose an algorithm that can utilize radiation-induced memory errors for real-time dosimetry. The algorithm is implemented in an Android platform using Android Studio IDE (Fig. 5(a)), targeted towards a Samsung Galaxy S5 testbed chosen as a median representative of recent Android devices. The application is written in the Android language, similar to the more prevalent Java. SD cards of the phone is 
used for the flash memory. The basic form of the algorithm developed is modeled in the flowchart of Fig. 5(b). The Android operating system imposes limits on how low-level an application can read, and as a result it is difficult to acquire raw FBC data from the SD card. Instead, the application can periodically attempt to read a given file. If the file becomes corrupted, the current application can so far only indicate that a potentially high dose has been received. Flash memory is not sensitive enough for personnel dosimetry, but such an application could be used for inexpensive sensitivearea monitoring.

\section{CONCLUSION}

In this paper we demonstrate that the state of the art Flash chips are sensitive enough to detect low radiation doses such as $100 \mathrm{rad}\left(\mathrm{SiO}_{2}\right)$. We find that $\mathrm{X}$-ray exposure causes significantly a higher fail bit count compared to gamma ray exposure, mostly likely due to dose enhancement effects associated with high-Z BEOL materials. In general, we find that the radiation-induced fail bit count varies between different pages, but the block-averaged failure count remains consistent among all the blocks. Thus, for dosimetry applications, average failure count on any block correlates with dose. We have also developed an algorithm for smart phone application that can potentially be used for real-time radiation dosimetry. Finally, we note that the observed extreme radiation sensitivity of these devices would be quite limiting for potential space applications.

\section{REFERENCES}

[1] E. Tochilin, N. Goldstein, and W. G. Miller, "Beryllium oxide as a thermoluminescent dosimeter," Health Phys., vol. 16, no. 1, pp. 1-7, Jan. 1969.

[2] A. Holmes-Siedle and L. Adams, "RADFET: A review of the use of metal-oxide-silicon devices as integrating dosimeters," Int. J. Radiat. Appl. Instrum. Part C Radiat. Phys. Chem., vol. 28, no. 2, pp. 235244, Jan. 1986.

[3] A. Jaksic, G. Ristic, M. Pejovic, A. Mohammadzadeh, C. Sudre, and W. Lane, "Gamma-ray irradiation and post-irradiation responses of high dose range RADFETs," IEEE Trans. Nucl. Sci., vol. 49, no. 3, pp. 1356-1363, Jun. 2002.

[4] M. Brucoli et al., "Floating gate dosimeter suitability for acceleratorlike environments," IEEE Trans. Nucl. Sci., vol. 64, no. 8, pp. 20542060, Aug. 2017.

[5] N. G. Tarr, K. Shortt, Y. Wang, and I. Thomson, "A sensitive, temperature-compensated, zero-bias floating gate MOSFET dosimeter," IEEE Trans. Nucl. Sci., vol. 51, no. 3, pp. 1277-1282, Jun. 2004.

[6] E. Wagner, R. Sorom, and L. Wiles, "Radiation monitoring for the masses," Health Phys., vol. 110, no. 1, p. 37-44, Jan. 2016.

[7] E. S. Snyder, P. J. McWhorter, T. A. Dellin, and J. D. Sweetman, "Radiation response of floating gate EEPROM memory cells," IEEE Trans. Nucl. Sci., vol. 36, no. 6, pp. 2131-2139, Dec. 1989.

[8] M. J. Kay, M. J. Gadlage, A. R. Duncan, D. Ingalls, A. Howard, and T. R. Oldham, "Effect of accumulated charge on the total ionizing dose response of a NAND flash memory," IEEE Trans. Nucl. Sci., vol. 59, no. 6, pp. 2945-2951, Dec. 2012.

[9] D. N. Nguyen, S. M. Guertin, G. M. Swift, and A. H. Johnston, "Radiation effects on advanced flash memories," IEEE Trans. Nucl. Sci., vol. 46, no. 6, pp. 1744-1750, Dec. 1999.

[10] N. Z. Butt and M. Alam, "Modeling single event upsets in Floating Gate memory cells," in Proc. 2008 IEEE International Reliability Physics Symposium, 2008, pp. 547-555.
[11] S. Gerardin et al., "Radiation effects in flash memories," IEEE Trans. Nucl. Sci., vol. 60, no. 3, pp. 1953-1969, Jun. 2013.

[12] S. Gerardin and A. Paccagnella, "Present and future non-volatile memories for space," IEEE Trans. Nucl. Sci., vol. 57, no. 6, pp. 30163039, Dec. 2010.

[13] M. Bagatin, G. Cellere, S. Gerardin, A. Paccagnella, A. Visconti, and S. Beltrami, "TID sensitivity of NAND flash memory building blocks," IEEE Trans. Nucl. Sci., vol. 56, no. 4, pp. 1909-1913, Aug. 2009.

[14] L. Z. Scheick, P. J. McNulty, and D. R. Roth, "Dosimetry based on the erasure of floating gates in the natural radiation environments in space," IEEE Trans. Nucl. Sci., vol. 45, no. 6, pp. 2681-2688, Dec. 1998.

[15] M. W. Savage, M. J. Gadlage, M. Kay, J. D. Ingalls, and A. Duncan, "Extreme value analysis in flash memories for dosimetry applications," IEEE Trans. Nucl. Sci., vol. 60, no. 6, pp. 4275-4280, Dec. 2013.

[16] D. M. Fleetwood, P. S. Winokur, and J. R. Schwank, "Using laboratory X-ray and cobalt-60 irradiations to predict CMOS device response in strategic and space environments," IEEE Trans. Nucl. Sci., vol. 35, no. 6, pp. 1497-1505, Dec. 1988.

[17] Y. Cai, S. Ghose, Y. Luo, K. Mai, O. Mutlu, and E. F. Haratsch, "Vulnerabilities in MLC NAND flash memory programming: experimental analysis, exploits, and mitigation techniques," in 2017 IEEE International Symposium on High Performance Computer Architecture (HPCA), 2017, pp. 49-60.

[18] D. M. Fleetwood, P. S. Winokur, L. J. Lorence, W. Beezhold, P. V. Dressendorfer, and J. R. Schwank, "The response of MOS devices to dose-enhanced low-energy radiation," IEEE Trans. Nucl. Sci., vol. 33, no. 6, pp. 1245-1251, Dec. 1986.

[19] T. R. Oldham and J. M. McGarrity, "Comparison of ${ }^{60}$ Co response and 10-keV X-ray response in MOS capacitors," IEEE Trans. Nucl. Sci., vol. 30, no. 6, pp. 4377-4381, Dec. 1983.

[20] M. R. Shaneyfelt, D. M. Fleetwood, J. R. Schwank, and K. L. Hughes, "Charge yield for cobalt-60 and 10-keV X-ray irradiations of MOS devices," IEEE Trans. Nucl. Sci., vol. 38, no. 6, pp. 1187-1194, Dec. 1991.

[21] C. M. Dozier, D. M. Fleetwood, D. B. Brown, and P. S. Winokur, "An evaluation of low-energy X-ray and cobalt-60 irradiations of MOS transistors," IEEE Trans. Nucl. Sci., vol. 34, no. 6, pp. 1535-1539, Dec. 1987.

[22] R. Micheloni, S. Aritome, and L. Crippa, "Array architectures for 3-D NAND flash memories," Proc. IEEE, vol. 105, no. 9, pp. 1634-1649, Sep. 2017.

[23] M. Danek, H. Bamnolker, R. Humayun, and J. Gao, "Tungsten for wordline applications," US20160233220A1, 11-Aug-2016.

[24] K. Tokunaga, J. Sel, M. Gunji-Yoneoka, and T. Pham, "Full metal gate replacement process for NAND flash memory," US9129854B2, 08Sep-2015.

[25] A. Dasgupta, D. M. Fleetwood, R. A. Reed, R. A. Weller, and M. H. Mendenhall, "Effects of metal gates and back-end-of-line materials on x-ray dose in $\mathrm{HfO}_{2}$ gate oxide," IEEE Trans. Nucl. Sci., vol. 58, no. 6, pp. 3139-3144, Dec. 2011. 